\title{
Neonatal progeria: increased ratio of progerin to lamin A leads to progeria of the newborn
}

\author{
Janine Reunert ${ }^{1}$, Rüdiger Wentzell ${ }^{2}$, Michael Walter ${ }^{3}$, Sibylle Jakubiczka ${ }^{4}$, Martin Zenker ${ }^{4}$, Thomas Brune ${ }^{4}$, \\ Stephan Rust ${ }^{5,6}$ and Thorsten Marquardt ${ }^{\star, 1,6}$
}

\begin{abstract}
Hutchinson-Gilford progeria syndrome (HGPS) is an important model disease for premature ageing. Affected children appear healthy at birth, but develop the first symptoms during their first year of life. They die at an average age of 13 years, mostly because of myocardial infarction or stroke. Classical progeria is caused by the heterozygous point mutation c.1824C $>T$ in the LMNA gene, which activates a cryptic splice site. The affected protein cannot be processed correctly to mature lamin $\mathrm{A}$, but is modified into a farnesylated protein truncated by 50 amino acids (progerin). Three more variations in LMNA result in the same mutant protein, but different grades of disease severity. We describe a patient with the heterozygous LMNA mutation c.1821G $>$ A, leading to neonatal progeria with death in the first year of life. Intracellular lamin A was downregulated in the patient's fibroblasts and the ratio of progerin to lamin A was increased when compared with HGPS. It is suggestive that the ratio of farnesylated protein to mature lamin A determines the disease severity in progeria.
\end{abstract}

European Journal of Human Genetics (2012) 20, 933-937; doi:10.1038/ejhg.2012.36; published online 14 March 2012

Keywords: neonatal progeria; HGPS; LMNA; progerin

\section{INTRODUCTION}

More than a century after the first description of the first progeria patients by Hutchinson and Gilford, ${ }^{1,2}$ researchers succeeded in identifying the genetic defect of the disease in the LMNA gene (MIM number 150330), ${ }^{3,4}$ which codes for the nuclear proteins lamin A and lamin C. Lamins are structural components of the nuclear lamina and furthermore responsible for the nuclear stability. Mutations in lamins cause a number of diseases, the so-called laminopathies, which include mandibuloacral dysplasia (MIM number 248370), Emery-Dreifuss muscular dystrophy (MIM number 181350), dilated cardiomyopathy 1A (MIM number 115200), Dunnigan-type familial partial lipodystrophy type 2 (MIM number 151660), limb-girdle muscular dystrophy type 1B (MIM number 159001), Charcot-Marie-Tooth disease (MIM number 605588) and Hutchinson-Gilford progeria syndrome (HGPS, MIM number 176670). Children with HGPS do not present a progeroid phenotype at birth, but develop typical symptoms during their first year of life. They die from cardiovascular disease at an average age of 13 years. The most common mutation leading to HGPS is a heterozygous de novo point mutation in exon 11 (c.1824C > T; p.Gly608Gly). This mutation activates a cryptic splice donor site, which is responsible for the excision of the C-terminal part of exon $11,{ }^{4}$ including the cleavage site of the zinc metalloprotease ZMPSTE24, an important enzyme in lamin A processing. ${ }^{5}$ The incomplete posttranslational modification of prelamin $\mathrm{A}$, the precursor of lamin $\mathrm{A}$, results in a truncated but farnesylated protein (progerin), which lacks 50 amino acids in the C-terminal part. This leads to an abnormal membrane association of the mutant protein throughout the cell cycle. ${ }^{6,7}$ Presence of progerin in the cell results in abnormal morphology of the nucleus, including nuclear blebbing, ${ }^{8}$ disrupted interactions with other nuclear envelope proteins (eg, nesprin and emerin), heterodimerization with wild-type lamin A isoforms ${ }^{9}$ and clustering of nuclear pores. ${ }^{10}$

The classical heterozygous c. $1824 \mathrm{C}>\mathrm{T}$ mutation is not the only genetic variation, which causes usage of the same alternative cryptic splice site in exon 11. To date, three more heterozygous mutations in LMNA lead to the same truncated protein (c.1821G $>$ A (p.Val607Val), c.1822G > A (p.Gly608Ser) and c. $1968+1 \mathrm{G}>\mathrm{A}),{ }^{11,12}$ but they do not result in the same level of disease severity. Codon 608 is affected in the classical c.1824C $>\mathrm{T}$ variant and also in c.1822G $>\mathrm{A}$. The mutations c. $1968+1 \mathrm{G}>\mathrm{A}$ and $\mathrm{c} .1821 \mathrm{G}>\mathrm{A}$ lead to a more severe phenotype compared with classical HGPS. Common to both patients was a higher expression of progerin, exceeding the amount of mature lamin $\mathrm{A}$ and a higher ratio of progerin to lamin A. ${ }^{12}$

The manuscript describes a patient with severe neonatal progeria and death at 3 months of age caused by the c.1821G $>$ A mutation in LMNA. In contrast to classical HGPS, expression of normal lamin A generated from the unaffected allele was severely reduced, giving new insights into the pathogenesis of premature ageing.

\section{MATERIALS AND METHODS}

Cell culture

A skin biopsy was taken from the patient and controls after informed consent of the parents. Cell lines with the classical c.1824C $>$ T mutation were obtained from the Progeria Research foundation (HGADFN127, HGADFN167, HGADFN003). Fibroblasts were cultured in Dulbecco's modified Eagle medium (PAA, Cölbe, Germany) supplemented with 10\% fetal calf serum (Invitrogen, Darmstadt, Germany) and 1\% L-glu-pen-strep (PAA).

\footnotetext{
${ }^{1}$ Universitätsklinikum Münster, Klinik für Kinder- und Jugendmedizin - Allgemeine Pädiatrie, Münster, Germany; ${ }^{2}$ Lukaskrankenhaus, Klinik für Kinder und Jugendliche, Neuss, Germany; ${ }^{3}$ Department of Laboratory Medicine and Pathobiochemistry, Campus Benjamin Franklin, Charité-Universitätsmedizin Berlin, Berlin, Germany; ${ }^{4}$ Institute of Human Genetics, University Hospital Magdeburg, Magdeburg, Germany; ${ }^{5}$ Leibniz-Institut für Arterioskleroseforschung, Münster, Germany

*Correspondence: Professor Dr T Marquardt, Klinik für Kinder- und Jugendmedizin - Allgemeine Pädiatrie, Albert-Schweitzer-Campus 1, Gebäude A1, 48149 Münster, Germany. Tel: +49 251835 8519; Fax: +49 251835 6085; E-mail: marquat@uni-muenster.de

6 These authors contributed equally to this work.

Received 24 August 2011; revised 20 December 2011; accepted 31 January 2012; published online 14 March 2012
} 


\section{Cell isolation}

Fibroblasts at $80-90 \%$ confluence were washed with phosphate-buffered saline (PBS) and trypsinized. Cell pellets were washed in PBS, and after centrifugation (5 min, $500 \mathrm{~g}$, room temperature), resuspended in HEPES-sucrose buffer $(0.2 \mathrm{M}$ sucrose, $0.02 \mathrm{M}$ HEPES, pH 7.3) containing protease inhibitors (Complete; Roche, Mannheim, Germany; $10 \mu \mathrm{g} / \mathrm{ml}$ pepstatin A, $10 \mu \mathrm{g} / \mathrm{ml}$ PMSF) for western blot analysis.

\section{Mutation analysis}

All exons of LMNA, including their flanking intronic sequences and the transcript (RefSeq NG_008692.1, NM_170707.2), were amplified by PCR. Genomic DNA of the patient and the parents was isolated from EDTA blood with the QIAmp DNA mini kit (Qiagen, Hilden, Germany) according to the manufacturer's protocol. cDNA of the patient was synthesized using MMLV Superscript III Reverse Transcriptase (Invitrogen) with oligo(dT) primers (Fermentas, St Leon-Rot, Germany) from total RNA extracted from fibroblasts, using the RNeasy Kit from Qiagen according to the manufacturer's protocol. Primers for amplification of the cDNA were designed using the Primer Express Software (PerkinElmer Life Science, Applied Biosystems, Foster City, CA, USA). Because of the length of the transcript, two primer pairs were designed, one amplifying the first part (c.-58-c.1038) and one amplifying the second part (c.988-c.2032). Sequences of primers used to amplify exons 1-12 (copied from www.dmd.nl/LMNA_primers.html, Seidman Laboratory, last accessed 8 July 2011) and both parts of the cDNA are listed in the Supplementary Material. All mutations and single-nucleotide polymorphisms (SNPs) were named according to the current Human Genomic Variant Society nomenclature.

\section{Cloning of cDNA sequences}

cDNA of the patient was prepared as described above and cloned into a TOPO TA cloning vector (TOPO TA cloning, Invitrogen). After amplification in bacteria, multiple clones were sequenced.

\section{SDS-PAGE and western blot}

For preparation of the nuclear fraction of whole-cell lysates, fibroblast cell pellets resuspended in HEPES-sucrose buffer were homogenized with a douncer and centrifugated for $15 \mathrm{~min}, 930 \mathrm{~g}$ and $4{ }^{\circ} \mathrm{C}$. The pelleted nuclei were solubilized in HEPES-sucrose buffer. Protein concentration was determined with Bradford protein assay and adjusted with loading buffer to $0.5 \mu \mathrm{g} / \mu \mathrm{l}$. Afterwards, the samples were heated at $95^{\circ} \mathrm{C}$ for $5 \mathrm{~min}$. Nuclear proteins $(2 \mu \mathrm{g})$ were loaded onto the SDS-PAGE. PageRuler protein ladder (SM0671, Fermentas) was used as molecular weight standard.

After separation by SDS-PAGE, the proteins were transferred electrophoretically to a PVDF membrane (Immobilon-P; Millipore, Schwalbach/Ts, Germany) and incubated with a polyclonal antibody for lamin A/C (sc-6215; Santa Cruz Biotechnology Inc., Heidelberg, Germany) diluted 1:2000 in PBST (PBS with $0.1 \%$ Tween) overnight at $4{ }^{\circ} \mathrm{C}$. After incubation with an HRPconjugated antibody anti-goat (P0449; Dako, Hamburg, Germany) diluted 1:5000 in PBST for $45 \mathrm{~min}$ at room temperature, the blot was developed for visualization of lamin A, lamin $\mathrm{C}$ and progerin, using the BM Chemiluminescence Western Blotting Substrate (POD; Roche) and high-performance chemiluminescence film (Amersham, GE Healthcare, Munich, Germany). Quantification of progerin and lamin A was done using the ImageJ software. ${ }^{13}$

\section{Gene expression analysis}

Quantitative real-time (qRT)-PCR was performed using an Applied Biosystems Prism 7900HT sequence detection system (Applied Biosystems, Darmstadt, Germany).

Primers were designed using the Primer Express Software (PerkinELmer Life Science). Primer sequences and location can be found in Supplementary Table S1, and Supplementary Figures S2 and S3. The assay for each sample was performed in 384-well plates with $25 \mathrm{ng}$ of reversed-transcribed RNA and SYBR Green PCR Master Mix (Eurogentec, Cologne, Germany) at a final volume of $12 \mu \mathrm{l}$. Results were evaluated using cyclophilin as the invariant control gene.

\section{RESULTS}

\section{Clinical features}

The boy (Figure 1a) was born as the third child of non-consanguineous parents after 34 weeks of pregnancy. Birth weight was $2190 \mathrm{~g}$ (40th percentile), body length $49 \mathrm{~cm}$ (85th percentile), head circumference $32 \mathrm{~cm}$ (40th percentile) and Apgar score 8/9/10. The skin was translucent and pergament-like blood vessels were clearly visible (Figure $1 \mathrm{~b}$ ); palpation of the skin revealed a strikingly stiff subcutaneous tissue. The patient had wide-open fontanelles and the cranial sutures were well palpable. The clavicula was truncated (Figure 1c). He had frontal bulging, hypoplastic low-set ears and hypertelorism. There were microand retrognathia, arthrogryposis with contractures of the fingers (Figure 1d) and talipes calcaneus on both sides (Figure 1e). Ultrasonic investigation of the brain revealed dilated lateral ventricles with a slightly bulging fontanel. These both normalized over time without any intervention. Mild splenomegaly was noted. Postpartal episodes of apnea were treated with caffeine citrate. Arterial hypertension was present. The patient showed few spontaneous movements. Enteral tube feeding was necessary. Paracetamol was given repeatedly as pain medication.

The patient developed progressive hypercapnic respiratory failure, which was predominantly due to restricted thoracic compliance related to the progressively stiff subcutaneous tissue.

Palliative care with oral morphine was instituted and the patient died at the age of 84 days during an episode of a mild respiratory infection.

\section{Mutation analysis}

Sequencing of the LMNA gene revealed a number of heterozygous variations in the patient's DNA (Table 1). Most of these mutations are known SNPs and were found in the father's DNA as well. Exon 11 carried the heterozygous mutation c.1821G $>$ A, which was not found in any of the parents. This mutation is just three

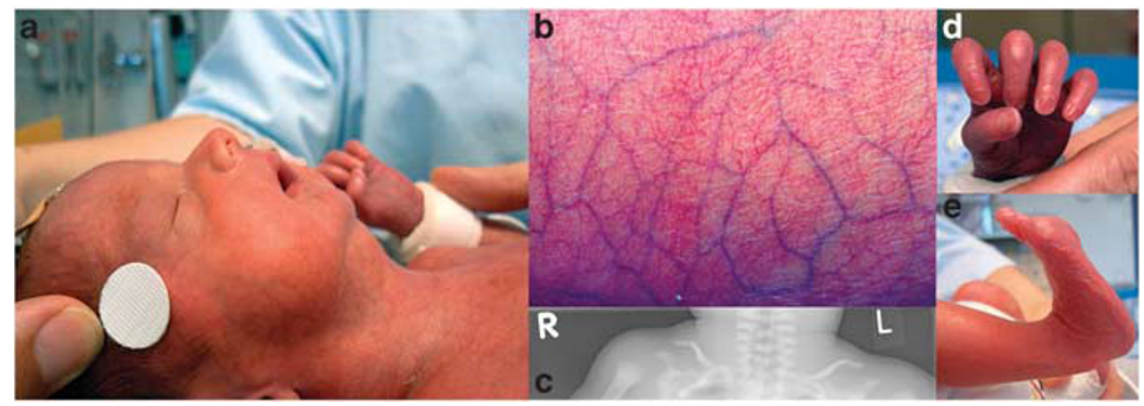

Figure 1 Photograph and X-ray of patient $\mathrm{N}$ and his skin (a, b), X-ray of the truncated clavicula (c), photograph of the foot and hand (d, e). 
Table 1 LMNA sequence alterations found in patient N, SNP rsID's included

\begin{tabular}{|c|c|c|c|}
\hline \multicolumn{4}{|l|}{ Exonic } \\
\hline c. $861 \mathrm{~T}>\mathrm{C}$ & p.Ala287Ala & Exon 5 & rs538089 \\
\hline c. $1338 \mathrm{~T}>\mathrm{C}$ & p.Asp446Asp & Exon 7 & rs505058 \\
\hline c. $1821 \mathrm{G}>\mathrm{A}$ & p.Val607Val & Exon 11 & Moulson et all ${ }^{12}$ \\
\hline \multicolumn{4}{|l|}{ Intronic } \\
\hline c. $639+56 \mathrm{G}>\mathrm{T}$ & - & Intron 3 & rs1126442 \\
\hline c. $639+73 \mathrm{~T}>\mathrm{C}$ & - & Intron 3 & rs1126443 \\
\hline c. $1157+16 \mathrm{G}>\mathrm{A}$ & - & Intron 7 & rs534807 \\
\hline c. $1380+142 G>A$ & - & Intron 8 & rs476000 \\
\hline
\end{tabular}

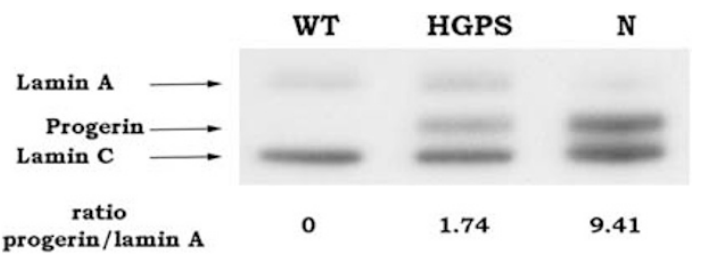

Figure 2 Western blot analysis with a lamin $A / C$ antibody in a healthy control (WT), a HGPS patient (HGPS) and patient $N(N)$. The ratio of progerin to lamin $\mathrm{A}$ in patient $\mathrm{N}$ exceeded the ratio in classical HGPS.

nucleotides upstream of the classical HGPS mutation c.1824C $>\mathrm{T}$ (Supplementary Figure S1A). It does not result in an amino acid substitution, but activates the same cryptic splice site as in classical HGPS, confirming the findings reported by Moulson et al. ${ }^{12}$ PCR of the first part of the cDNA (c.-58-c.1038) generated a normal-size fragment, whereas PCR of the second part (c.988-c.2032) generated two fragments of different size identical to classical HGPS (Supplementary Figure S1C). Sequencing of the patient's cDNA revealed the same sequence as in HGPS (Supplementary Figure S1B), indicating that progerin is also produced as a consequence of the heterozygous c.1821C $>$ T mutation.

\section{Cloning of cDNA sequences}

To examine whether the c.1821G $>$ A mutation found in the patient was maternally or paternally derived, cDNA sequences of the patient were cloned into a TOPO TA cloning vector and amplified in bacteria. Multiple clones were sequenced. Both alleles could be detected, one of them carried all variations found in patient N (Table 1), including c.1821G $>$ A; the other allele carried the wild-type sequence (data not shown), indicating the c.1821G $>$ A mutation and the other sequence variations were localized on the paternally inherited allele.

\section{SDS-PAGE and western blot}

For western blot analysis, nuclear proteins of fibroblasts of a healthy control, patient $\mathrm{N}$, and a patient with the typical c.1824C $>\mathrm{T}$ (HGPS) mutation were used. Signals of lamin A, lamin C and the truncated progerin (in HGPS and patient $\mathrm{N}$ ) can be seen in a western blot using a lamin A/C antibody (Figure 2). The progerin signal of patient $\mathrm{N}$ was more intense than that of the HGPS patient (HGPS2). The amount of lamin A was severely reduced compared with classical HGPS, and the ratio of progerin to mature lamin $\mathrm{A}$ in patient $\mathrm{N}$ was 9.41, the highest ratio observed in progeria patients yet.
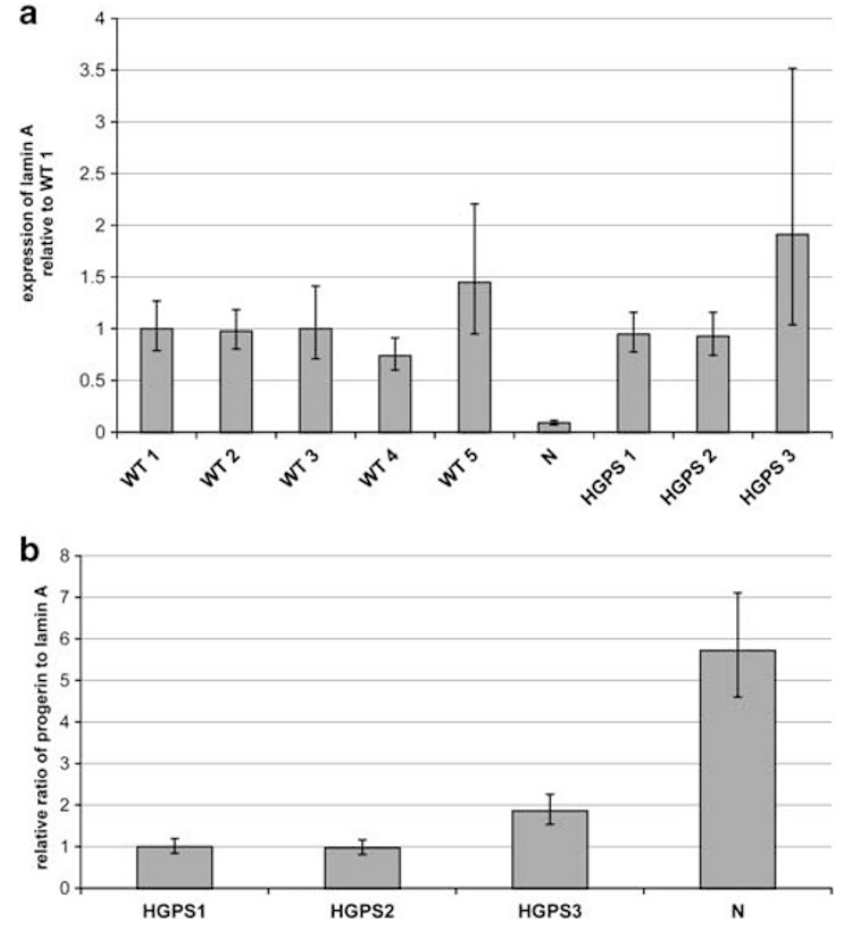

Figure $3 L M N A$ expression analysis in healthy controls (WT), progeria patients (HGPS) and patient $N(N)$. Expression of full-length lamin $A$ relative to WT 1 (a) and the ratio of progerin to full-length lamin $A$ relative to HGPS 1 (b) are shown.

\section{Gene expression analysis}

LMNA expression was analyzed by RT-PCR in primary fibroblasts of healthy controls $(n=5)$, progeria patients with the classical c. $1824 \mathrm{C}>\mathrm{T}$ mutation $(n=3)$ and patient $\mathrm{N}$ (Figure 3). Expression of lamin A, measured by RT-PCR with a primer pair that exclusively amplificates lamin A (primer 'lamin_A1'), was equal in HGPS and control fibroblasts. In contrast, the expression of lamin A in patient $\mathrm{N}$ was only about $10 \%$ when compared with controls (Figure $3 \mathrm{a}$ ). The amounts of progerin mRNA (primer 'progerin') ${ }^{14}$ in HGPS patients and patient $\mathrm{N}$ were comparable, whereas the ratio of progerin to mature lamin A was about six-fold higher in patient $\mathrm{N}$ (Figure $3 \mathrm{~b}$ ). The sum of lamin A, lamin $\mathrm{C}$ and progerin mRNA, as measured by RT-PCR with a primer pair located in exons 7 and 8 ('lamin_A3'), was slightly increased in HGPS when compared with healthy controls, and reduced to about $50 \%$ in patient $\mathrm{N}$ (data not shown).

\section{DISCUSSION}

HGPS is a disease that usually is not clinically recognized at birth, as the full phenotype becomes apparent after the first year of life, although at least one case of neonatal progeria in children carrying the classical p.Gly608Gly mutation has been described. ${ }^{15}$ Neonatal progeria is usually known as Wiedemann-Rautenstrauch syndrome (WRS, MIM number 264090), which is characterized by a progeroid phenotype already apparent after birth. In contrast to HGPS, WRS patients do not have a mutation in the LMNA gene, ${ }^{11}$ the genetic defect is still unknown.

The neonatal progeroid phenotype of our patient suggested a more severe disease than in classical HGPS. After sequencing the LMNA gene, the classical mutation leading to HGPS in exon 11 could be excluded. However, several heterozygous sequence variations were found in the gene, one of them just three nucleotides upstream of 
the classical c. $1824 \mathrm{C}>\mathrm{T}$ mutation. Except for this mutation (c.1821G $>$ A), all other sequence variations were also found in the father's DNA. A variety of diseases are caused by paternally derived autosomal dominant mutations, especially single-base substitutions (eg, in achondroplasia). ${ }^{16}$ As already shown by Eriksson et al ${ }^{4}$ and D'Apice et al, ${ }^{17}$ also the classical HGPS mutation usually arises in the paternal germline. Only one exception with a maternal-derived mutation was described by Wuyts et al, ${ }^{18}$ explained by a gonadal mosaicism in the mother. By sequencing multiple cDNA clones, all of the variations, including c.1821G $>A$, were found on the same allele, proving that the pathogenic mutation in exon 11 was paternally derived and that the patient also carried one wild-type lamin A allele.

After sequencing the cDNA, it was obvious that the identical cryptic splice site was used as in classical progeria, leading to exactly the same progerin protein. However, in contrast to HGPS, the patient described here had a much more severe clinical phenotype, resulting in early death at an age of 84 days. The severe phenotype of patient $\mathrm{N}$ is similar to that observed in patient I in Moulson et al, ${ }^{12}$ who had the same pathogenic mutation in LMNA and died after 26 days. Unlike patient $\mathrm{I}$, our patient did not show intrauterine growth retardation or a cleft palate.

In contrast to normal lamin $\mathrm{A}$, progerin is truncated and farnesylated. It has been shown in mice, that the farnesyl group of progerin is essential for the development of progeria. Homozygous lamin C-only mice $\left(\mathrm{Lmna}{ }^{\mathrm{LCO} / \mathrm{LCO}}\right)$, carrying a mutant allele that leads exclusively to lamin $\mathrm{C}$, demonstrate that a lack of mature lamin A results in no clinical phenotype, normal emerin targeting and only minimal alterations in nuclear shape. ${ }^{19}$ In these cells, no mature lamin A and no farnesylated lamin A isoform is present. Two mouse models were created expressing an unfarnesylated form of progerin..$^{20,21}$ The Cterminal -caax motif was changed from - csim to - ssim $\left(\mathrm{Lmna}^{\mathrm{nHG} /+}\right)$ in one model, and to $-\mathrm{csm}\left(\mathrm{Lmna}^{\mathrm{csm} /+}\right)$ in the other one. $\mathrm{Lmna}^{\mathrm{nHG} /+}$ mice showed a mild progeria-like disease phenotype, whereas the Lmna $^{\text {csm/+ }}$ mice developed normally. ${ }^{20,21}$

Furthermore, even the homozygous $\mathrm{Lmna}^{\mathrm{csm} / \mathrm{csm}}$ mice did not present a progeria-like disease phenotype. ${ }^{21}$ Although they neither express lamin A nor lamin C, their body weight curves, survival curves and adipose tissue stores were indistinguishable from wild-type littermate control mice. In contrast, lamin $\mathrm{A} / \mathrm{C}$ knockout mice died within 8 weeks, ${ }^{22}$ suggesting that non-farnesylated progerin can substitute for the survival-relevant functions of lamin A/C. The mild progeroid phenotype in $\mathrm{Lmna}^{\mathrm{nHG} /+}$ mice might be because of the missing free cysteine in the C-terminus of progerin, which might somehow protect from the disease. ${ }^{21}$

If only farnesylated prelamin A is generated inside the cell because of a loss of ZMPSTE24 activity, the resulting disorder named restrictive dermatopathy (RD, MIM number 275210) has a phenotype much more severe than HGPS. Children with RD usually die either in utero or within days after birth. Non-farnesylated prelamin A as sole lamin A isoform might be sufficient to prevent severe cell damage. Lmna ${ }^{\mathrm{nPLAO} / \mathrm{nPLAO}}$ mice, expressing only non-farnesylated prelamin A, appeared normal for the first 20 weeks of life, but developed a cardiomyopathy and died earlier than healthy mice (median survival in female mice 49.5 weeks, in male mice 38.5 weeks). ${ }^{23}$

By contrast, the presence of farnesylated progerin next to mature lamin A in classical HGPS disturbs the nuclear integrity and results in the typical progeroid phenotype with death in early adolescence.

$\mathrm{Lmna}^{\mathrm{LCO} / \mathrm{LCO}}, \mathrm{Lmna}^{\mathrm{csm} / \mathrm{csm}}, \mathrm{Lmna}^{\mathrm{nHG} /+}$ and $\mathrm{Lmna}^{\text {nPLAO/nPLAO }}$ mice, HGPS and RD differ by the amount of farnesylated protein in the cell and show an immense difference in disease severity. In the patient described here, as well as in the patient described by Moulson et $a l,{ }^{12}$ the amount of farnesylated protein was increased compared with HGPS, but was not the exclusive form of lamin A as it is in RD. Also, the phenotype and the clinical features of these patients indicate an intermediate state between HGPS and RD.

These findings suggest a dose effect of the farnesylated protein on cell structure and organization, with an increasing amount of farnesylated protein leading to a more severe phenotype. The accumulation of farnesylated protein might damage the cell in a way that it cannot maintain its organization. Possibly, the stability of the nucleus collapses because of the incorrect distribution or deficient oligomerization of the farnesylated lamins.

Western blots and qPCR experiments showed clearly that the amount of normal lamin A generated from the healthy allele was severely reduced in patient $\mathrm{N}$ (Figures 2 and 3). As this change is not only seen at the protein level, but is already present at the level of the mRNA, expression of the normal lamin A allele is downregulated in patient N (Figure 3). Such a severe downregulation of lamin A in an HGPS patient has never been described before. In contrast, the amounts of progerin transcript in the patient and in the HGPS controls were comparable, indicating that not only the absolute amount of progerin determines disease severity, but the ratio of progerin to normal lamin A. A possible explanation would be an overexpression of the tumor suppressor AIMP3 that has been shown to lead to selective degradation of mature lamin A, but not of lamin C, prelamin A or progerin. ${ }^{24}$ However, as the lamin A amount is already reduced at the mRNA level, it is reasonable to assume that also a reduced amount of lamin A protein is synthesized and that AIMP3 does not affect the lamin A quantity in our patient. Confirming this suggestion, RT-PCR analysis of AIMP3 in fibroblasts of patient $\mathrm{N}$ did not show a significant overexpression when compared with healthy controls and HGPS fibroblasts (data not shown).

Differences between the progerin to lamin A ratios at the levels of the mRNA and the protein in patient $\mathrm{N}$ occur through the decreased amount of normal lamin A. Moulson et al ${ }^{12}$ also detected differences between the progerin to lamin A ratio at mRNA and protein levels in patient I. They assumed that progerin might be more stable than mature lamin A, possibly due to the farnesyl residue still attached to the protein. An increased ratio of progerin to mature lamin A was also determined in a fibroblast cell line from a 9-year-old HGPS patient, when compared with fibroblasts of younger HGPS patients. ${ }^{25}$ Related to the increased progerin to lamin A ratio, Columbaro et al ${ }^{25}$ assumed a worsening with patient age. We suggest that an increase of the ratio of progerin to mature lamin A increases the severity of the clinical phenotype explaining the severe phenotype of patient $\mathrm{N}$ directly after birth. In patients $\mathrm{I}$ and $\mathrm{N}$, the higher progerin to lamin $\mathrm{A}$ ratio obviously lead to a more severe phenotype when compared with classical HGPS. Both patients (I and N) died during an infection; therefore, the life expectancy of both patients cannot be correlated exclusively with the progerin to lamin A ratio. Patient TB in Moulson et $a l^{12}$ had a higher ratio of progerin to lamin A as patient I, but showed a milder phenotype and got 3 years old. We cannot estimate the lifetime of patients I and $\mathrm{N}$ without the infection.

To date, four different mutations in LMNA are known to lead to the activation of the cryptic splice site in exon 11 (Figure 4). Moulson et $a l^{12}$ used the MaxENT scoring method to determine the usage frequency of this splice site..$^{26,27}$ The score for the typical HGPS mutation c. $1824 \mathrm{C}>\mathrm{T}$ is 8.56 , whereas the score for the c.1821G $>\mathrm{A}$ variation is 9.88 . The score for the wild-type sequence is 8.07 . These findings indicate that the alternative splice site in patient $\mathrm{N}$ might be used more frequently than in classical HGPS. As the normal lamin A allele is downregulated in patient $\mathrm{N}$ (Figure 3), we cannot confirm or 


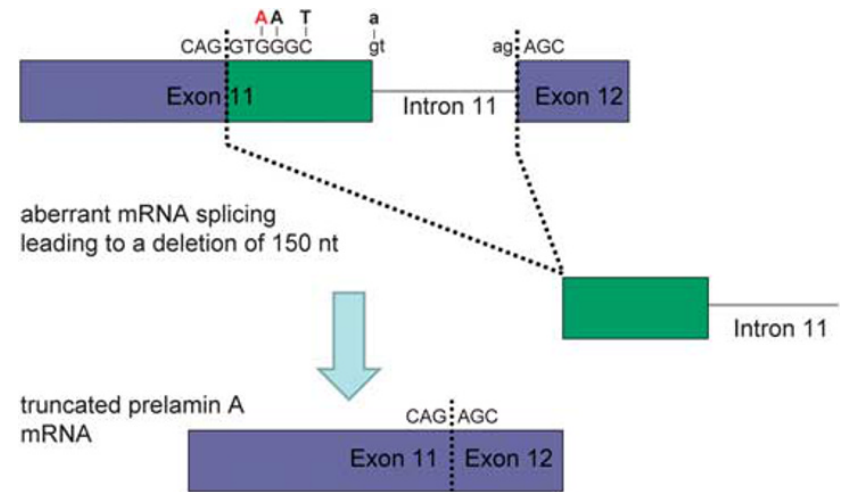

Figure $4 \mathrm{~A}$ schematic model for lamin $\mathrm{A}$ and progerin splicing. Four mutations in $L M N A$ leading to the usage of the identical cryptic splice site, resulting in a truncated, farnesylated protein. The mutation c.1821G $>$ A leading to the neonatal progeroid phenotype in patient $\mathrm{N}$ is tagged in red.

exclude a higher frequency of progerin splicing when compared with HGPS. If both alleles would be equally transcribed and the mutant allele would be spliced to progerin to $100 \%$, the ratio of progerin to lamin A would reach a maximum of 1:1. Instead, we observed a progerin to lamin A ratio of 6:1. It is known that there are splicing silencer and enhancer regions in exon, as well as in intron sequences, varying between different tissues and individuals, ${ }^{28}$ which could contribute to splicing differences in patient $\mathrm{N}$ and $\mathrm{I}$.

Moreover, variable ratios between lamin A and another splice variant, lamin $\mathrm{C}$, have been observed earlier, both at protein as well as mRNA levels, ${ }^{29,30}$ and may occur tissue-specific and cell-cycle dependent. ${ }^{31,32}$ However, the exact mechanisms have not yet been elucidated.

In summary, the heterozygous c.1821G $>\mathrm{A}$ mutation in LMNA leads to neonatal progeria with a much more severe phenotype than classical HGPS. HGPS, neonatal progeria and RD have an increasing ratio of the farnesylated lamin $\mathrm{A}$ isoform to mature lamin $\mathrm{A}$, correlating to an increased disease severity and suggesting a doseeffect model of progeria. Current treatment trials in HPGS aim to reduce the amount of prenylation of progerin, a concept that is well supported by the dose-effect model.

\section{CONFLICT OF INTEREST}

The authors declare no conflict of interest.

\section{ACKNOWLEDGEMENTS}

We thank the patients' family for their support and their open mindedness regarding the research on premature ageing diseases, and the Progeria Research Foundation for providing HGPS fibroblasts.

1 Gilford H: Progeria: a form of senilism. Practitioner 1904; 73: 188-217.

2 Hutchinson J: Congenital absence of hair and mammary glands with atrophic condition of the skin and its appendages in a boy whose mother had been almost totally bald from alopecia areata from the age of six. Med Chirurg Trans 1886; 69: 36.

3 De Sandre-Giovannoli A, Bernard R, Cau P et al: Lamin A truncation in HutchinsonGilford progeria. Science 2003; 300: 2055.
4 Eriksson M, Brown WT, Gordon LB et al: Recurrent de novo point mutations in lamin A cause Hutchinson-Gilford progeria syndrome. Nature 2003; 423: 293-298.

5 Pendás AM, Zhou Z, Cadiñanos J et al: Defective prelamin A processing and muscular and adipocyte alterations in Zmpste24 metalloproteinase-deficient mice. Nat Genet 2002; 31: 94-99.

6 Cao K, Capell BC, Erdos MR, Djabali K, Collins FS: A lamin A protein isoform overexpressed in Hutchinson-Gilford progeria syndrome interferes with mitosis in progeria and normal cells. Proc Natl Acad Sci USA 2007; 104: 4949-4954.

7 Dechat T, Shimi T, Adam SA et al: Alterations in mitosis and cell cycle progression caused by a mutant lamin A known to accelerate human aging. Proc Natl Acad Sci USA 2007; 104: 4955-4960.

8 Goldman RD, Shumaker DK, Erdos MR et al: Accumulation of mutant lamin A causes progressive changes in nuclear architecture in Hutchinson-Gilford progeria syndrome. Proc Natl Acad Sci USA 2004; 101: 8963-8968.

9 Delbarre E, Tramier M, Coppey-Moisan M, Gaillard C, Courvalin JC, Buendia B: The truncated prelamin $\mathrm{A}$ in Hutchinson-Gilford progeria syndrome alters segregation of Atype and B-type lamin homopolymers. Hum Mol Genet 2006; 15: 1113-1122.

10 Coutinho HD, Falcão-Silva VS, Gonçalves GF, da Nóbrega RB: Molecular ageing in progeroid syndromes: Hutchinson-Gilford progeria syndrome as a model. Immun Ageing 2009; 6: 4.

$11 \mathrm{Cao} \mathrm{H}$, Hegele RA: LMNA ist mutated in Hutchinson-Gilford progeria (MIM176670) but not in Wiedemann-Rautenstrauch progeroid syndrome (MIM 264090). J Hum Genet 2003; 48: 271-274.

12 Moulson CL, Fong LG, Gardner JM et al: Increased progerin expression associated with unusual LMNA mutations causes severe progeroid syndromes. Hum Mutat 2007; 28: 882-889

13 Rasband WS. ImageJ, U. S. National Institutes of Health, Bethesda, Maryland, USA, http://imagej.nih.gov/ij/ 1997-2011.

14 Scaffidi P, Misteli T: Lamin A-dependent nuclear defects in human aging. Science 2006; 312: 1059-1063.

15 Sevenants L, Wouters C, De Sandre-Giovannoli A et al: Tight skin and limited joint movements as early presentation of Hutchinson-Gilford progeria in a 7-week-old infant. Eur J Pediatr 2005; 164: 283-286.

16 Crow JF: The origins, patterns and implications of human spontaneous mutation. Nat Rev Genet 2000; 1: 40-47.

17 D’Apice MR, Tenconi R, Mammi I, van den Ende J, Novelli G: Paternal origin of LMNA mutations in Hutchinson-Gilford progeria. Clin Genet 2004; 65: 52-54.

18 Wuyts W, Biervliet M, Reyniers E, D'Apice MR, Novelli G, Storm K: Somatic and gonadal mosaicism in Hutchinson-Gilford progeria. Am J Med Genet A 2005; 135: 66-68.

19 Fong LG, Ng JK, Lammerding J et al: Prelamin A and lamin A appear to be dispensable in the nuclear lamina. J Clin Invest 2006; 116: 632-634.

20 Yang SH, Andres DA, Spielmann HP, Young SG, Fong LG: Progerin elicits disease phenotypes of progeria in mice whether or not it is farnesylated. J Clin Invest 2008; 118: 3291-3300.

21 Yang SH, Chang SY, Ren S et al: Absence of progeria-like disease phenotypes in knockin mice expressing a non-farnesylated version of progerin. Hum Mol Genet 2011; 20: 436-444

22 Sullivan T, Escalante-Alcalde $\mathrm{D}$, Bhatt $\mathrm{H}$ et al: Loss of A-type lamin expression compromises nuclear envelope integrity leading to muscular dystrophy. J Cell Biol 1999; 147: 913-919.

23 Davies BS, Barnes 2nd RH, Tu Y et al: An accumulation of non-farnesylated prelamin A causes cardiomyopathy but not progeria. Hum Mol Genet 2010; 19: 2682-2694.

24 Oh YS, Kim DG, Kim G et al: Downregulation of lamin A by tumor suppressor AIMP3/ p18 leads to a progeroid phenotype in mice. Aging Cell 2010; 9: 810-822.

25 Columbaro M, Capanni C, Mattioli E et al: Rescue of heterochromatin organization in Hutchinson-Gilford progeria by drug treatment. Cell Mol Life Sci 2005; 62 : 2669-2678.

26 Eng L, Coutinho G, Nahas S et al: Nonclassical splicing mutations in the coding and noncoding regions of the ATM gene: maximum entropy estimates of splice junction strengths. Hum Mutat 2004; 23: 67-76.

27 Yeo G, Burge CB: Maximum entropy modeling of short sequence motifs with applications to RNA splicing signals. J Comput Biol 2004; 11: 377-394.

28 Conboy JG, Dubchak I: Alternative splicing in humans. Encyclopedia of Genetics, Genomics, Proteomics and Bioinformatics. London: Wiley, 2005.

29 Broers JL, Raymond Y, Rot MK, Kuijpers H, Wagenaar SS, Ramaekers FC: Nuclear Atype lamins are differentially expressed in human lung cancer subtypes. Am J Pathol 1993; 143: 211-220.

30 Machiels BM, Broers JL, Raymond $Y$ et al: Abnormal A-type lamin organization in a human lung carcinoma cell line. Eur J Cell Biol 1995; 67: 328-335.

31 Miranda M, Chacón MR, Gutiérrez $\mathrm{C}$ et al: LMNA mRNA expression is altered in human obesity and type 2 diabetes. Obesity (Silver Spring) 2008; 16: 1742-1748.

32 Pugh GE, Coates PJ, Lane EB, Raymond Y, Quinlan RA: Distinct nuclear assembly pathways for lamins $A$ and $C$ lead to their increase during quiescence in Swiss 3T3 cells. J Cell SCi 1997; 110: 2483-2493.

Supplementary Information accompanies the paper on European Journal of Human Genetics website (http://www.nature.com/ejhg) 